\title{
Agile Development and UX Design: Towards Understanding Work Cultures to Support Integration
}

\author{
Jennifer Ferreira \\ University of Calgary, Calgary AB T2N 1N4, Canada \\ jen.ferreira@ucalgary.ca
}

\begin{abstract}
Organisations are investing heavily into enterprise system infrastructure. With a move to agility and Agile software development, there is an increasing need for understanding how Agile developers and User Experience (UX) designers work together in practice. This paper outlines the current approaches to investigating the combination of Agile development and UX design and indicates a direction for future research that could benefit integration across the cross-functional teams required for enterprise software development.
\end{abstract}

Keywords: Agile development, User Experience design, integration, studies of work practice, work group culture.

\section{Introduction}

As enterprise environments embrace agility and Agile software development, there is also a need for understanding how User Experience (UX) designers 1 fit into Agile projects. The benefits of Agile development combined with UX design are well documented in the literature. For practitioners, the advantages include an improved product 12, better quality of the user experience 3, increased team confidence [4], team morale [56], devotion and satisfaction [7]. For academic researchers, the combination of Agile development and UX design is of interest as part of a broader, on-going effort for better integration of HumanComputer Interaction (HCI) into software engineering 81910.

There are, however, non-trivial problems that Agile development and UX design practitioners have to work through in order to achieve these benefits. There are problems reported regarding the timing and scheduling of Agile development and UX design tasks. Such issues include involving users [1112] and incorporating their feedback back into the development effort 1314, coordinating between the activities of the UX designers, Agile developers and other non-Agile teams [1115] and performing usability testing in the context of other Agile development tests, e.g. unit testing and acceptance testing [16]. Further,

${ }^{1}$ User Experience $(U X)$ design refers to a collection of approaches that design the users' experience with the software by setting out to understand users and how they will use the software, and iteratively refining the design. 
UX designers working with Agile development teams find that time for up-front UX design and user research is short 81116 17/18, and in some cases there is no allocated time for up-front research 3 .

Both Agile development and UX design aim to build quality software, but despite their common concern, each approaches development from a different perspective. While Agile methods mainly describe activities addressing code creation (e.g. 19]), UX design methods describe activities for designing the product's interaction with a user [20]. There is little guidance about integrating these two perspectives, and still few detailed accounts providing a close scrutiny of Agile development and UX design being combined in practice. While the growing body of literature continues to focus on process descriptions and recommended techniques, the day-to-day work involved and the many and varied settings in which the techniques and processes are applied, remain largely unexplored.

\section{Processes, Techniques and Situatedness}

\subsection{Processes and Techniques that Establish and Maintain Focus}

The combination of UX design with Agile development helps practitioners maintain focus on important aspects of software development. While Agile development focuses on creating working software, UX design focuses on creating a usable design that may or may not be in the form of working software. A central concern is to support the weaknesses of one with the strengths of the other. That is, Agile development is seen to lack an awareness of usability issues, with little guidance for how and when designers contribute to the process (e.g. [15]). According to Patton [4, eXtreme Programming (XP) allowed the team to deliver on time, however, they were still not satisfying the end user. Only by adding the techniques of Usage-Centered Design could they focus on user goals and focus on what was required to build. UX design approaches lack a structured approach to transforming designs into working software and, therefore, little guidance on how developers are involved. Besides a focus on end-users practitioner accounts show a variety of ways in which UX design supports Agile development, such as supporting planning and prioritisation [21], bridging communications between stakeholders 22]. Wilcox et al. 23] also point out the bridging effect of design: "Design, in this and many more cases, acted as a middle-man between the highlevel vision and bottom-up innovations under development." Therefore, the two are seen as complementary approaches that, used together, improve the outcome of the software development effort. Examples in the literature combine established Agile approaches, e.g., XP, or Scrum, with established design approaches, e.g., Usage-Centered Design [24], Usability Engineering [25]. We also find examples of well-known HCI techniques such as personas [26] and scenarios [27] being used on Agile projects.

\subsection{Dealing with a Piecemeal Approach to Development}

Agile values discourage up-front planning activities for software design, i.e., upfront design of the code [28, which often affects time allocated for UX design 
on Agile projects. When Agile projects allocate little time for performing UX design before implementation begins, and allow requirements to change during development, the holistic view of the product is compromised and in some instances seen to be missing 31213 . It is understood that UX designers require enough time at the outset of the project to perform user research and sketch out a coherent design. Practitioners have been found to do some UX design up front in order to get the development effort started [29:9] and in fact consider up-front UX design more acceptable than up-front code design [30]. Advice is generally to have designers remain ahead of the developers, so that they have enough time to design for what is coming ahead and evaluate what has already been implemented - beginning with a stage before implementation begins, i.e,. "Cycle Zero" 313] or "Sprint Zero" 32, then interleaving the activities of UX design work with implementation work throughout the duration of the project (e.g. 31]). To fit with the time-boxed Agile iterations, usability techniques are often adapted to fit within shorter timescales. Lee and McCrickard [13] suggest performing lightweight usability testing as part of the acceptance testing process. This approach is successful when usability tests are smaller, more focused and performed more often. Sy [3] suggests adjusting the timing and granularity of user research.

\subsection{The Situated Nature of Practice}

Another strand of research moves away from a focus on process descriptions and specific techniques and investigates the combination of Agile development and UX design by engaging with the settings in which practitioners work. These studies are still rare and, as a result, assumptions about practice and the factors that shape how developers and designers work together are largely unexplored. The few existing studies that have taken account of organisational settings have observed practitioners working in everyday contexts. These include the framework for integrating Agile development and user-centred design proposed by Chamberlain et al. 8], the discovery of the importance of identity and vision in successful UX design by Kollmann et al. 33. and the forms of collaboration between developers and designers by Brown et al. 34.

Such studies are an important contribution to understanding how Agile development and UX design practitioners work together, as there is evidence that both developers [35] and designers [36] deviate from prescribed processes in practice. Whether prescribed processes are followed depends on the relation between the individuals and the environment in which they work — which can be referred to as situatedness. Suchman introduces the term situated action to emphasise that 'every course of action depends in essential ways upon its material and social circumstances' 37. p. 50]. Considering Agile development and UX design work as situated action has the implication that process descriptions, on their own, are not adequate for understanding practice [38]. Complexity in real work settings arises from different balances of power and different levels of influence the designers and developers may have on determining how they work. Therefore, a focus on the day-to-day negotiations of order that take into consideration 
the wider setting in which the practitioners are embedded allows a better understanding of work arrangements, dependencies and mechanisms that make the integration and coordination of work possible. Further, rather than considering the Agile teams as self-contained units separated from their wider organisational setting, we have to take into consideration the settings in which they carry out their work, i.e. we have to take into account that they may be reliant on other parties for getting their work done and that their wider organisational setting has consequences for how they get their work done.

\section{Towards Understanding Work Group Cultures}

While there is advice in the literature for how Agile developers and UX designers can mesh their activities (e.g. 313]), empirical evidence based on observations of what Agile developers and UX designers actually do suggests that integration relies on recurring efforts in engaging other individuals for their input [39]. This does not follow any of the suggested process models in the current literature. Instead, progress requires the involvement of those with decision-making power, or expertise, when the work demands it. This could include engaging with developers, designers or other members of the organization, depending on how decision-making power is distributed, or the type of expertise required to perform the particular task at hand.

Previous research on contextual factors influencing the integration of $\mathrm{Ag}$ ile development with UX design has emphasised the importance of values and assumptions underlying decision-making [40. This approach assumes that underlying the decisions concerning how Agile development and UX design should work together in the organisation, are values and assumptions that motivate those decisions - a value being a belief about what ought to be [41, p. 25]. This relationship between decisions, values and assumptions has been studied by other researchers as levels of organisational culture, the visible aspects of which are observable in its artefacts 42. While the research that gave rise to the importance of values and assumptions was not aimed at understanding organisational culture, elements that can be associated with culture showed up in the analysis of the data and were significant in explaining how and why the Agile developers and UX designers in each team were working together in the ways that had been observed. Explicating values and assumptions gave insights into differing views on how "best" to create quality software and how "best" to create software combining the skills of UX designers and Agile developers.

Agile developers and UX designers do what is required in order to get their job done, by maintaining focus and coordinating between their tasks, expecting certain behaviours from others, maintaining mutual awareness, negotiating progress and engaging with each other. Their work in each setting depends on the values endorsed by the organisations in which the developers and designers are embedded. Two views emerged: (1) the best way of developing quality software is by keeping the Agile developers and UX designers separate, and (2) the best way of developing quality software is via the Agile developers and UX 
designers working closely together. Those teams embedded in settings endorsing similar values, also had similar experiences in carrying out their work. Further details appear in Ferreira et al. [40]. The significance of articulating these values and assumptions allows them to be discussed and compared, so (in)forming part of a wider, ongoing debate about how UX design and Agile development should be combined. Second, the analysis shows that the integration of UX design and Agile development in practice can not be characterised solely by adaptations of different processes and techniques. Rather, the empirically-based results demonstrate clearly that the nature of UX/Agile practice is characterised strongly in terms of the values of the organisations in which they are embedded. Therefore, instead of merely prescribing processes and adding techniques, improving practice requires also a shift in focus to the explication of contextual values.

One way of taking this discussion further, would be to analyse practice in terms of culture. Agile software development research has addressed culture on a global level (e.g. 43]) and at the organisational level (e.g. 44]). Cultures endorse and constrain the behaviours of individuals embedded in organisations [45] and may therefore provide a useful analytical lens for studying Agile development and UX design in practice. Vaughan [46] has shown that culture does not necessarily have to extend to an entire organisation. By studying work group culture the focus can be the "set of solutions produced by a group of people to meet specific problems posed by the situation that they face in common" [46, p. 64]. As the success of enterprise systems rely on the integration of various organisational entities [4], a sympathetic understanding of the cultures endorsed by each entity could aid an understanding of how to better support their interactions.

\section{Concluding Remarks}

This paper indicates a direction for future research that could benefit integration across the cross-functional teams required for enterprise software development - that of studying work group culture. Current research on the combination of Agile development and UX design places an emphasis on processes and tools, while research addressing practice in the settings in which it unfolds remains scarce. A move towards understanding work group cultures endorsed by the entities involved in enterprise software development could aid an understanding of how to better support their interactions, beyond prescribing processes and techniques that are rarely followed in practice.

\section{References}

1. Meszaros, G., Aston, J.: Adding usability testing to an agile project. In: Proceedings of the Conference on AGILE 2006, pp. 289-294. IEEE Computer Society, Washington, DC (2006)

2. Singh, M.: U-SCRUM: An Agile methodology for promoting usability. In: Agile 2008: Proceedings of the Agile 2008 Conference, Toronto, Ontario, Canada, August 4-8, pp. 555-560. IEEE Computer Society, Los Alamitos (2008) 
3. Sy, D.: Adapting usability investigations for Agile user-centered design. Journal of Usability Studies 2(3), 112-132 (2007)

4. Patton, J.: Designing Requirements: Incorporating Usage-Centered Design into an Agile SW Development Process. In: Wells, D., Williams, L. (eds.) XP 2002. LNCS, vol. 2418, pp. 1-12. Springer, Heidelberg (2002)

5. Frank, A., Hartel, C.: Feature teams collaboratively building products from ready to done. In: Agile Conference, AGILE 2009, pp. 320-325 (August 2009)

6. Hussain, Z., Lechner, M., Milchrahm, H., Shahzad, S., Slany, W., Umgeher, M., Wolkerstorfer, P.: Agile User-Centered Design Applied to a Mobile Multimedia Streaming Application. In: Holzinger, A. (ed.) USAB 2008. LNCS, vol. 5298, pp. 313-330. Springer, Heidelberg (2008), doi:10.1007/978-3-540-89350-9_22

7. Lindström, H., Malmsten, M.: User-centred design and agile development: Rebuilding the swedish national union catalogue. The Code\{4\}Lib Journal 5, 12-15 (2008)

8. Chamberlain, S., Sharp, H., Maiden, N.A.M.: Towards a Framework for Integrating Agile Development and User-Centred Design. In: Abrahamsson, P., Marchesi, M., Succi, G. (eds.) XP 2006. LNCS, vol. 4044, pp. 143-153. Springer, Heidelberg (2006)

9. Fox, D., Sillito, J., Maurer, F.: Agile methods and user-centered design: How these two methodologies are being successfully integrated in industry. In: Agile 2008: Proceedings of the Agile 2008 Conference, Toronto, Ontario, Canada, August 4-8, pp. 63-72. IEEE Computer Society, Los Alamitos (2008)

10. Memmel, T., Gundelsweiler, F., Reiterer, H.: Agile human-centered software engineering. In: HCI 2007: Proceedings of 21st BCS HCI Group Conference, University of Lancaster, UK, pp. 167-175. British Computer Society (September 2007)

11. Detweiler, M.: Managing UCD within Agile projects. Interactions 14(3), 40-42 (2007)

12. Wolkerstorfer, P., Tscheligi, M., Sefelin, R., Milchrahm, H., Hussain, Z., Lechner, M., Shahzad, S.: Probing an Agile usability process. In: CHI 2008: CHI 2008 Extended Abstracts on Human Factors in Computing Systems, pp. 2151-2158. ACM, New York (2008)

13. Lee, J.C., McCrickard, S.: Towards extreme(ly) usable software: Exploring tensions between usability and Agile software development. In: Agile 2007: Proceedings of the Agile 2007 Conference, Washington, DC, USA, August 13-17, pp. 59-71. IEEE Computer Society, Los Alamitos (2007)

14. McInerney, P., Maurer, F.: UCD in Agile projects: Dream team or odd couple? Interactions 12(6), 19-23 (2005)

15. Budwig, M., Jeong, S., Kelkar, K.: When user experience met agile: A case study. In: Proceedings of the 27th International Conference Extended Abstracts on $\mathrm{Hu}-$ man Factors in Computing Systems, CHI 2009, pp. 3075-3084. ACM, New York $(2009)$

16. Sy, D., Miller, L.: Optimizing Agile user-centered design. In: CHI 2008: CHI 2008 Extended Abstracts on Human Factors in Computing Systems, pp. 3897-3900. ACM, New York (2008)

17. Ambler, S.W.: Tailoring usability into Agile software development projects. In: Law, E., Hvannberg, E., Cockton, G. (eds.) Maturing Usability. Human-Computer Interaction Series, pp. 75-95. Springer, London (2008)

18. Ungar, J., White, J.: Agile user centered design: Enter the design studio - a case study. In: CHI 2008: CHI 2008 Extended Abstracts on Human Factors in Computing Systems, pp. 2167-2178. ACM, New York (2008)

19. Beck, K., Andres, C.: Extreme Programming Explained: Embrace Change, 2nd edn. Addison-Wesley, Boston (2004) 
20. Sharp, H., Rogers, Y., Preece, J.: Interaction Design: Beyond Human-Computer Interaction, 2nd edn. John Wiley \& Sons (2007)

21. Patton, J.: Finding the forest in the trees. In: Companion to the 20th Annual ACM SIGPLAN Conference on Object-oriented Programming, Systems, Languages, and Applications, OOPSLA 2005, pp. 266-274. ACM, New York (2005)

22. Broschinsky, D., Baker, L.: Using persona with XP at LANDesk Software, an Avocent company. In: Agile 2008: Proceedings of the Agile 2008 Conference, Toronto, Ontario, Canada, August 4-8, pp. 543-548. IEEE Computer Society, Los Alamitos (2008)

23. Wilcox, E., Nusser, S., Schoudt, J., Cerruti, J., Badenes, H.: Agile Development Meets Strategic Design in the Enterprise. In: Concas, G., Damiani, E., Scotto, M., Succi, G. (eds.) XP 2007. LNCS, vol. 4536, pp. 208-212. Springer, Heidelberg (2007)

24. Patton, J.: Hitting the target: Adding interaction design to Agile software development. In: OOPSLA 2002: OOPSLA 2002 Practitioners Reports, pp. 1-7. ACM, New York (2002)

25. Kane, D.: Finding a place for discount usability engineering in Agile development: Throwing down the gauntlet. In: ADC 2003: Proceedings of the 2003 Agile Development Conference, Salt Lake City, UT, USA, June 25-28, pp. 40-46. IEEE Computer Society, Los Alamitos (2003)

26. Haikara, J.: Usability in Agile Software Development: Extending the Interaction Design Process with Personas Approach. In: Concas, G., Damiani, E., Scotto, M., Succi, G. (eds.) XP 2007. LNCS, vol. 4536, pp. 153-156. Springer, Heidelberg (2007)

27. Obendorf, H., Finck, M.: Scenario-based usability engineering techniques in Agile development processes. In: CHI 2008: CHI 2008 Extended Abstracts on Human Factors in Computing Systems, pp. 2159-2166. ACM, New York (2008)

28. Fowler, M.: Is Design Dead? (2004) published online, http://martinfowler.com/articles/designDead.html\#id20933 (updated May 2004) (last accessed June 2011)

29. Ferreira, J., Aumann, Y., Biddle, R.: Up-Front Interaction Design in Agile Development. In: Concas, G., Damiani, E., Scotto, M., Succi, G. (eds.) XP 2007. LNCS, vol. 4536, pp. 9-16. Springer, Heidelberg (2007)

30. Ferreira, J., Noble, J., Biddle, R.: Agile development iterations and ui design. In: Agile 2007: Proceedings of the Agile 2007 Conference, Washington, DC, USA, August 13-17, pp. 50-58. IEEE Computer Society, Los Alamitos (2007)

31. Miller, L.: Case study of customer input for a successful product. In: Agile 2005: Proceedings of the 2005 Agile Conference, Denver, CO, USA, July 24-29, pp. 225234. IEEE Computer Society, Los Alamitos (2005)

32. Najafi, M., Toyoshiba, L.: Two case studies of user experience design and Agile development. In: Agile 2008: Proceedings of the Agile 2008 Conference, Toronto, Ontario, Canada, August 4-8. IEEE Computer Society, Los Alamitos (2008)

33. Kollmann, J., Sharp, H., Blandford, A.: The importance of identity and vision to user experience designers on agile projects. In: Proceedings of the 2009 AGILE Conference, Chicago, IL, USA, August 24-28, pp. 11-18. IEEE Computer Society (2009)

34. Brown, J., Lindgaard, G., Biddle, R.: Collaborative events and shared artefacts: Agile interaction designers and developers working toward common aims. In: AGILE Conference (AGILE), pp. 87-96 (August 2011)

35. Fitzgerald, B.: The use of systems development methodologies in practice: A field study. Info. Systems J. 7, 201-212 (1997) 
36. Gasson, S.: The reality of user-centered design. J. End User Comput. 11(4), 5-15 (1999)

37. Suchman, L.A.: Plans and Situated Actions: The problem of human-machine communication. Cambridge University Press, New York (1987)

38. Ferreira, J., Sharp, H., Robinson, H.: User experience design and agile development: Managing cooperation through articulation work. Software: Practice and Experience 41(9), 963-974 (2011)

39. Ferreira, J., Sharp, H., Robinson, H.: Agile development and user experience design integration as an on-going achievement in practice. Accepted to Agile 2012 to be held in Dallas, TX (2012)

40. Ferreira, J., Sharp, H., Robinson, H.: Values and Assumptions Shaping Agile Development and User Experience Design in Practice. In: Sillitti, A., Martin, A., Wang, X., Whitworth, E. (eds.) XP 2010. LNBIP, vol. 48, pp. 178-183. Springer, Heidelberg (2010), doi:10.1007/978-3-642-13054-0_15

41. Schein, E.H.: Organizational Culture and Leadership, 4th edn. Jossey-Bass, San Francisco (2010)

42. Schein, E.H.: Organizational culture. American Psychologist 45(2), 109-119 (1990)

43. Hossain, E., Bannerman, P.L., Jeffery, R.: Towards an understanding of tailoring scrum in global software development: a multi-case study. In: Proceedings of the 2011 International Conference on Software and Systems Process, ICSSP 2011, pp. 110-119. ACM, New York (2011)

44. Strode, D., Huff, S., Tretiakov, A.: The impact of organizational culture on agile method use. In: 42nd Hawaii International Conference on System Sciences, HICSS 2009, pp. 1-9 (January 2009)

45. Vaughan, D.: Signals and interpretive work: The role of culture in a theory of practical action. In: Cerulo, K.A. (ed.) Culture in Mind: Toward a Sociology of Culture and Cognition, pp. 28-54. Routledge, New York (2002)

46. Vaughan, D.: The Challenger Launch Decision: Risky technology, culture and deviance at NASA. The University of Chicago Press, Chicago and London (1996)

47. Nah, F.F.H., Lau, J.L.S., Kuang, J.: Critical factors for successful implementation of enterprise systems. Business Process Management Journal 7(3), 285-296 (2001) 